\title{
Structured association analysis leads to insight into Saccharomyces cerevisiae gene regulation by finding multiple contributing eQTL hotspots associated with functional gene modules
}

Ross E Curtis ${ }^{1,2}$, Seyoung Kim², John L Woolford $\mathrm{Jr}^{3}$, Wenjie $\mathrm{Xu}^{3}$ and Eric P Xing ${ }^{4^{*}}$

\begin{abstract}
Background: Association analysis using genome-wide expression quantitative trait locus (eQTL) data investigates the effect that genetic variation has on cellular pathways and leads to the discovery of candidate regulators. Traditional analysis of eQTL data via pairwise statistical significance tests or linear regression does not leverage the availability of the structural information of the transcriptome, such as presence of gene networks that reveal correlation and potentially regulatory relationships among the study genes. We employ a new eQTL mapping algorithm, GFlasso, which we have previously developed for sparse structured regression, to reanalyze a genome-wide yeast dataset. GFlasso fully takes into account the dependencies among expression traits to suppress false positives and to enhance the signal/noise ratio. Thus, GFlasso leverages the gene-interaction network to discover the pleiotropic effects of genetic loci that perturb the expression level of multiple (rather than individual) genes, which enables us to gain more power in detecting previously neglected signals that are marginally weak but pleiotropically significant.
\end{abstract}

Results: While eQTL hotspots in yeast have been reported previously as genomic regions controlling multiple genes, our analysis reveals additional novel eQTL hotspots and, more interestingly, uncovers groups of multiple contributing eQTL hotspots that affect the expression level of functional gene modules. To our knowledge, our study is the first to report this type of gene regulation stemming from multiple eQTL hotspots. Additionally, we report the results from in-depth bioinformatics analysis for three groups of these eQTL hotspots: ribosome biogenesis, telomere silencing, and retrotransposon biology. We suggest candidate regulators for the functional gene modules that map to each group of hotspots. Not only do we find that many of these candidate regulators contain mutations in the promoter and coding regions of the genes, in the case of the Ribi group, we provide experimental evidence suggesting that the identified candidates do regulate the target genes predicted by GFlasso.

Conclusions: Thus, this structured association analysis of a yeast eQTL dataset via GFlasso, coupled with extensive bioinformatics analysis, discovers a novel regulation pattern between multiple eQTL hotspots and functional gene modules. Furthermore, this analysis demonstrates the potential of GFlasso as a powerful computational tool for eQTL studies that exploit the rich structural information among expression traits due to correlation, regulation, or other forms of biological dependencies.

\footnotetext{
* Correspondence: epxing@cs.cmu.edu

${ }^{4}$ Machine Learning Department, Carnegie Mellon University, Pittsburgh, PA, USA

Full list of author information is available at the end of the article
} 


\section{Background}

Expression quantitative trait locus (eQTL) analysis has been widely used to understand how genetic variations in the genome perturb biological systems by altering cellular mRNA levels [1]. A typical eQTL study involves genotype data collected for genetic markers, such as single nucleotide polymorphisms (SNPs), along with microarray data for a population of individuals. These studies aim to identify genes whose expression level varies according to genetic variation. As both the genotype and gene expression data are collected at a genome-wide scale, structured, as opposed to the traditional trait-by-trait eQTL analysis must be employed to probe the complex interplay between the genome and phenome at a systems level rather than at the level of individual loci and genes. For example, genetic variation can perturb the expression of a gene, which then can affect the activity of other genes downstream in the same pathway. A mutation in a regulator, such as a transcription factor, can influence the expression of all of the regulator's target genes, leading to a pleiotropic effect. On the other hand, genetic variants at multiple different loci may jointly influence the expression of some genes, through an additive or an epistatic effect. Analyzing a genome-wide eQTL dataset allows us to discover the complex patterns of how genetic variants give rise to variation in expression level. At the same time, examining multiple genes or multiple traits jointly in a genome-wide analysis can give insight into the functional roles that genetic variants play in a biological system and can potentially lead to the discovery of new regulators in a region of associated SNPs.

Many eQTL datasets have been collected for various organisms, including yeast [2], mouse [3], human [4], and Arabidopsis [5], as well as for different diseases such as diabetes [6]. Additionally, different statistical approaches have been developed that go beyond traditional single-trait analysis to unravel the complex patterns of association between the genetic variants and expression levels. In particular, we have previously developed a new statistical paradigm for eQTL mapping called structured association mapping [7]. In this paper we employ a structured association mapping method called GFlasso, which leverages the full gene-expression network to guide a search for genotypes that influence genes whose expression levels are highly correlated [7]. To our knowledge, this method is the first that systematically exploits the full gene correlation network in eQTL analysis.

GFlasso finds associations between SNPs and subnetworks of correlated genes within the full network by exploiting the gene network, avoiding many of the fundamental limitations of previous methods. For example, performing association analysis using the PCA-based method on transformed traits sacrificed the interpretability of the results. Lirnet used gene expression levels averaged over genes within each cluster and then maps these averages to genetic loci [8]. In this case, however, the averaging operation can lead to the loss of information on the activity of individual genes, especially genes whose expression levels are negatively correlated. Although Zhu et al. [9] and other work by the same group of researchers took advantage of the gene network in eQTL analyses, they did so only as a post-processing step after finding eQTLs for each gene separately, rather than directly using the network during the search for eQTLs. It is only recently that methods to exploit the rich information in the gene expression network have been developed [7,10-12].

In this study, we use structured association mapping to reanalyze the yeast eQTL dataset available from Brem and Kruglyak [13] with a new focus on uncovering the genetic basis behind the coupled gene-expression traits. The dataset includes the genome-wide profiling of expression levels and SNPs for 112 recombinant progeny from two parent strains, a laboratory strain and a wild vineyard strain. We choose this particular dataset because it has been previously analyzed using different computational methods, providing a useful test bed for comparing structured association mapping with other methods. Since the method we use, GFlasso, leverages the gene network in eQTL analysis to combine information across correlated traits, it has the potential to achieve greater statistical power and discover relatively weak association signals that were missed in previous analyses. In fact, our analysis of the yeast eQTL dataset stemming from GFlasso provided new insights into the complex interaction between genetic variations and the transcriptome in yeast.

Many of the previous computational analyses of this same dataset reported regions in the genome, coined eQTL hotspots, which control the expression level of gene clusters that are highly enriched for a common function $[8,9]$. This suggested a coordinated genetic control of gene modules. Also, by examining the eQTL hotspot regions in the genome, these studies identified candidate regulators whose genetic variations lead to a perturbation of the gene cluster's gene expression levels.

While our structured association analysis rediscovers these previously reported eQTL hotspots and their regulators, we identify additional novel eQTL hotspots of biological significance. More interestingly, we find that many of these gene modules are associated with not one, but multiple eQTL hotspots. Although the presence of eQTL hotspots has been reported previously, to our knowledge our analysis is the first to find multiple eQTL hotspots that contribute to the same functional gene module. We perform in-depth bioinformatics analysis of three groups of these eQTL hotspots that we have uncovered. Based on the shared function of the genes perturbed by the eQTL hotspots in each group, we name the three groups the ribosome biogenesis (Ribi) group, the 
telomere group, and the retrotransposon group, and we suggest candidate regulators for each group of hotspots. Our bioinformatics analysis of each group of eQTL hotspots provides new insight into gene regulation in yeast. For example, in our analysis of the Ribi group, we find a coordinated regulation of ribosome biogenesis by multiple genomic loci on chromosomes II, V, and VII. We show experimentally that the expression levels of a subset of 43 of these target genes change when the $P B F 2$, $K A P 114, R A I 1$, and REI1 are knocked out. In our analysis of the telomere group, we discover candidate regulators (NUP2, RIF2, SIR3, YRF1) in four genomic regions on chromosome IV, X, and XII that likely play a coordinated role in telomere silencing. We identify mutations in the promoter and coding regions of the candidate regulators using the full genome sequences available from public databases to provide strong evidence that the candidate regulators are true regulators. Finally, in the retrotransposon group, we discover the coordinated effects of 17 retrotransposon insertions on the resulting expression signal for retrotransposons. We note that in all three modules, this coordinated effect could be epistatic or additive.

\section{Results}

We applied GFlasso to analyze a genome-wide eQTL dataset generated from a cross between the BY4617 (BY) strain (isogenic to yeast strain S288c) and the vineyard RM11-1a (RM) strain of Saccharomyces cerevisiae, baker's yeast [13]. The dataset consists of these two parent strains and 112 recombinant progeny. We considered the 1260 unique SNP markers on all 16 chromosomes, which cover nearly the entire genome at a resolution of about $20 \mathrm{~kb}$. We used these SNP markers to find associations to the mRNA expression levels for 5637 genes (genes with more than $30 \%$ missing values were excluded from analysis).

GFlasso assumes that a network for the gene-expression traits is available as prior knowledge, and GFlasso leverages this network in a structured sparse regression framework to identify associations between genetic loci and multiple traits that are tightly connected in the network. In our preprocessing step, we constructed a scale-free and modular network from the gene-expression data (see Methods for more detail) $[9,14]$. We used the resulting topological overlap matrix [14] as our gene-expression network. Once we estimated the parameters for association strengths using GFlasso (see Methods), we carried out an in-depth biological analysis. We have automated the full pipeline of our analysis and made it available with our distribution of the GenAMap software platform for genome-wide association (GWAS) and eQTL analysis $[15,16]$.

\section{Gene modules under regulation of common genetic loci}

We examined the eQTLs estimated by GFlasso for clusters of genes controlled by common genetic loci
(Figure 1). We divided up the genome into 428 genomic regions based on the linkage disequilibrium (LD) between the SNPs in this dataset (see in Additional file 1: Supplementary Methods). We define an eQTL module as all of the genes that map to the same genomic region. We define an eQTL hotspot as a genomic region whose eQTL module is greater than 40 genes. An eQTL hotspot and its corresponding eQTL module imply a pleiotropic effect of the genetic locus on co-regulated genes in a common pathway. We note that in our definition of an eQTL module, a gene could be a member of multiple eQTL modules, each associated with different eQTL hotspots. In a biological system, this corresponds to the situation of multiple contributing genetic loci, where the expression of the gene is affected by multiple trans-acting loci as well as a possible cis-acting locus through either an additive or an epistatic effect.

Although we found many eQTL modules, in this study we focus on those eQTL modules with greater than 40 genes, that is, only those that map to eQTL hotspots (in Additional file 1: Figure S1). We present an analysis of 22 such eQTL modules that vary in size from 42 to 722 genes. Ten of the 22 corresponding eQTL hotspots were novel discoveries in this dataset. The other $12 \mathrm{eQTL}$ hotspots overlapped with the 13 eQTL hotspots that had been reported in previous analyses of the same dataset; all 13 previous eQTL hotspots were recovered as two previously discovered eQTL hotspots were combined in our analysis $[8,9,17]$.

The common association that an eQTL hotspot has to all the genes in an eQTL module suggests that the region harbors regulators that influence the expression levels of the genes in the eQTL module. We list some candidate regulators located in cis to each eQTL hotspot in Table 1. All the genes within $20 \mathrm{~kb}$ from the eQTL hotspot are potential candidates, but because many genes are located in cis to each eQTL hotspot, we select transcription factors, genes in the eQTL module, and other genes involved in the pathway of the eQTL module to list here. In Table 1, we compare our results with those obtained from a computational analysis using a Bayesian network modeling approach [9], Lirnet [8], and known and possible regulators based on literature search [17]. In general, we found that the results were consistent between GFlasso and previous analyses. For example, in eQTL hotspot 4 located on chromosome III around $200 \mathrm{~kb}$, GFlasso found 62 genes in the eQTL module; five of these genes, MATALPHA1, MATALHPA2, PHO87, BUD5, and TAF2, are located in cis to this eQTL hotspot and therefore they are candidate regulators of the eQTL module. Consistent with our results, three previous analyses discussed MATALPHA1 as a regulator for genes in this eQTL module, and Lirnet additionally suggested MATALPHA2 and TBK1. As these candidate regulators lie in cis to this eQTL hotspot region, 


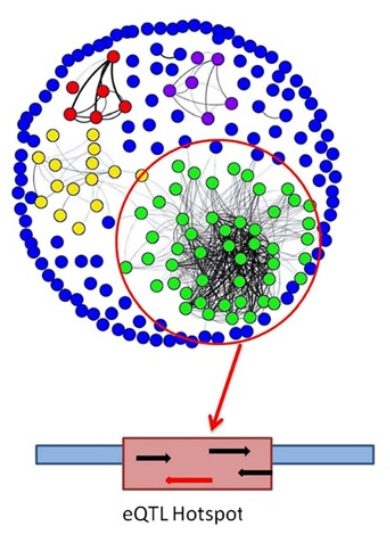

(a)

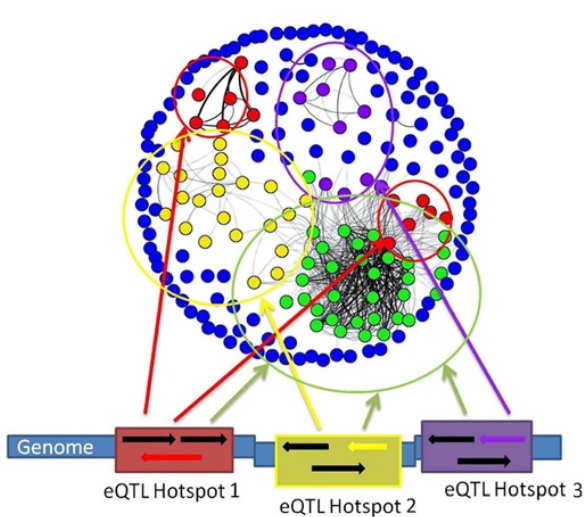

(b)

Figure 1 An illustration of our main results. Figure legend text (a) Previous analyses (e.g., [9,17,35]) of the yeast eQTL dataset reported eQTL hotspots, a module of multiple genes controlled by the same genomic locus. (b) In our GFlasso analysis of the same dataset, we not only found eQTL hotspots, but also discovered multiple contributing eQTL hotspots, where the same module of multiple genes is associated with multiple eQTL hotspots. This figure was created using GenAMap [16].

Table 1 The eQTL hotspots and their candidate regulators from GFlasso and other previous analyses

\begin{tabular}{|c|c|c|c|c|c|}
\hline eQTL Hotspot & eQTL module size & cis genes in eQTL module GFlasso & Yvert et al. [17] & Zhu et al. [9] & Lee et al. [8] \\
\hline *II:380000 & 106 & NRG1 TIP1 TAT1 TEC1 ECM33 & none & none & RDH54 SEC18 SPT7 \\
\hline *II:560000 & 722 & $\begin{array}{l}\text { AMN1 MAK5 CNS1 TBS1 TOS1 ARA1 } \\
\text { SUP45 CSH1 RPB5 SDS24 ENP1 REI1 }\end{array}$ & AMN1 MAK5 & $\begin{array}{l}\text { AMN1 CNS1 TBS1 TOS1 } \\
\text { ARA1 SUP45 CSH1 }\end{array}$ & $\begin{array}{c}\text { AMN1 CNS1 TOS2 ABD1 } \\
\text { PRP5 TRS20 }\end{array}$ \\
\hline *III:100000 & 225 & $\begin{array}{c}\text { LEU2 ILV6 NFS1 CIT2 PGS1 RER1 } \\
\text { HIS4 FRM2 KCC4 }\end{array}$ & LEU2 & $\begin{array}{l}\text { LEU2 ILV6 NFS1 CIT2 } \\
\text { MATALPHA1 }\end{array}$ & LEU2 ILV6 PGSI \\
\hline *III:200000 & 62 & $\begin{array}{c}\text { MATALPHA1 MATALPHA2 PHO87 } \\
\text { BUD5 TAF2 }\end{array}$ & MATALPHA1 & MATALPHA1 & $\begin{array}{c}\text { MATALPHA1 MATALPHA2 } \\
\text { TBK1 }\end{array}$ \\
\hline IV:1500000 & 46 & YRF1-1 YDR539W YDR541C & - & - & - \\
\hline *V:110000 & 45 & URA3 NPP2 & URA3 & URA3 & URA3 NPP2 PAC2 \\
\hline V:350000 & 618 & RPS24A RPS8B RTT105 & - & - & - \\
\hline V:420000 & 405 & LCP5 NSA2 & - & - & - \\
\hline V:460000 & 42 & YER138C UBP5 RTR1 & - & - & - \\
\hline VII:50000 & 350 & RAl1 TAD1 KAP114 & - & - & - \\
\hline *VIII:110000 & 147 & $\begin{array}{c}\text { GPA1 YAP3 ERG11 LAG1 SHO1 } \\
\text { ETP1 YLF2 LEU5 }\end{array}$ & GPA1 & GPA1 & GPA1 STP2 NEM1 \\
\hline$X: 20000$ & 48 & YJL225C VTH2 FSP2 REE1 & - & - & - \\
\hline XII:610000 & 53 & TOP3 & - & - & - \\
\hline *XII:680000 & 185 & HAP1 NEJ1 SSP120 & HAP1 & HAP1 & HAP1 NEJ1 GSY2 \\
\hline XII:780000 & 44 & REC102 PEX30 FKS1 GAS2 & - & - & - \\
\hline *XII:1070000 & 54 & YRF1-4 YRF1-5 YLR464W YLR462W & SIR3 & YRF1-4 YRF1-5 YLR464W & SIR3 HMG2 ECM7 \\
\hline *XII:70000 & 76 & MDM1 & none & none & ARG81 TAF13 CAC2 \\
\hline *XIV:490000 & 448 & $\begin{array}{c}\text { SAL1 TOP2 MKT1 THO2 MSK1 TPM1 } \\
\text { LAT1 SWS2 }\end{array}$ & none & SAL1 TOP2 & TOP2 MKT1 MSK1 \\
\hline XIV:550000 & 45 & COG6 YIP3 HDA1 & - & - & - \\
\hline$X V: 90000$ & 85 & $\begin{array}{l}\text { ZEO1 RFC4 HM11 INO4 NDJ1 } \\
\text { SKM1 HAL9 }\end{array}$ & - & - & - \\
\hline *XV:180000 & 406 & PHM7 ATG19 WRS1 RFC4 & none & PHM7 & PHM7 ATG19 BRX1 \\
\hline *XV:59000 & 120 & $\begin{array}{l}\text { LSC1 YOR131C RAS1 INP53 } \\
\text { OST2 PIN2 }\end{array}$ & CAT5 & none & CAT5 ADE2 ORT1 \\
\hline
\end{tabular}

* denotes a previously discovered hotspot. 
the genetic variation in this region may directly influence the activity or expression of these regulators, which then influence the expression of other genes in the eQTL module.

\section{Multiple contributing eQTL hotspots with pleiotropic effects on common eQTL modules}

In order to see if the genes in an eQTL module controlled by an eQTL hotspot share function, we performed a gene ontology (GO) enrichment analysis using the GO Slim annotation from the Saccharomyces Genome Database (SGD) [18]. As can be seen in Table 2, many of the eQTL modules were significantly enriched for common GO categories, indicating the genes in the eQTL module share function. For example, the eQTL module associated with the eQTL hotspot at XII:680 kb is enriched for genes annotated to lipid metabolic process $(\mathrm{GO}$ category, $p$-value $=1.3 \mathrm{e}-14)$ in biological process (GO category type), and the eQTL module associated with the XIV:450 kb eQTL hotspot is enriched for translation (GO Category, $p$-value $=2.3 \mathrm{e}-28$ ) in biological process (GO category type). For the previously reported eQTL hotspots, our results were consistent with those from previous GO enrichment analysis.

In addition, we performed enrichment analyses on each of the eQTL modules using two knockout datasets $[19,20]$ and four transcription factor binding datasets [21-24]. The results from these enrichment analyses are available in the Additional file 1: Supplementary Methods and the Additional file 2: Supplementary GFlasso Results. From the transcription factor target enrichment results, we found that genes in each eQTL module involved in the same GO process were also generally enriched for the binding of a common transcription factor and a knockout perturbation as has been shown before [9].

Interestingly, as we considered the results from the $\mathrm{GO}$ enrichment analysis and the transcription factor and knockout analyses, we noticed groups of eQTL modules that were enriched for the same GO annotations, transcription factor binding, and knockout signatures. For example, the eQTL modules associated to eQTL hotspots II:560 kb, V:350 kb, V:420 kb, and VII:50 kb were all significantly enriched for ribosome biogenesis and PBF2 binding. Furthermore, as shown in Figure 2, these four eQTL modules had a large overlap in member genes. This suggests that a large number of genes in the four eQTL modules are regulated in-part by each of these different eQTL hotspots. As the eQTL modules share the common GO annotation of ribosome biogenesis (Ribi), we call this group of eQTL hotspots the Ribi group (Figure 3 ).

Additionally, we found another group of eQTL modules, associated with eQTL hotspots IV:1500 kb, $\mathrm{X}: 20 \mathrm{~kb}, \mathrm{XII}: 780 \mathrm{~kb}$, and XII:1070 kb, all significantly enriched for helicase activity. Again, the eQTL modules corresponding to these eQTL hotspots shared a large fraction of member genes (Figure 2), and many of the genes that are shared by these four eQTL modules were annotated with common GO terms (Figure 3). Since our bioinformatics analysis of this group of eQTL modules revealed that they are involved in telomere activity, we name this group of eQTL hotspots the telomere group. As we considered the telomere group and the Ribi group, we noticed that the genes regulated by three or more of the eQTL hotspots in the group had a higher GO enrichment than genes that were associated with only one or two eQTL hotspots (Figure 3). Thus, in subsequent analysis we focus primarily on the genes associated with at least three of the four eQTL hotspots.

In addition to the Ribi and telomere groups of eQTL hotspots, we searched for other groups of eQTL hotspots with an overlap of more than 20 genes in the corresponding eQTL modules. Using this criterion, we identified one additional group of eQTL modules mapping to eQTL hotspots at V:460 kb, VIII:110 kb, and $\mathrm{XV}: 90 \mathrm{~kb}$ (Table 2). Although we did not find a common $\mathrm{GO}$ enrichment for these eQTL modules, further analysis revealed that many of the genes are involved in retrotransposon biology. Thus, we name this group the retrotransposon group.

We note that only one of the eQTL hotspots associated with each of the Ribi, telomere, and retrotransposon groups has been found in previous analyses of the same dataset, while all of the other eQTL hotspots associated to the groups of eQTL modules are novel discoveries from our GFlasso analysis. Thus, to our knowledge, these groups of eQTL hotspots with a common eQTL module have not been found in any of the previous analyses of this dataset. Thus, our GFlasso and bioinformatics analysis provides new insight into the genetic control of gene expression in a cell, especially the pleiotropic effect of multiple contributing genetic loci.

We compared the GFlasso results to those from a previous study which looked for epistatic interactions in this dataset [25]. After replicating the previous analysis, we found few overlaps between the results from the two analyses; the two studies share only one gene affected by the same two genomic bins (see in Additional file 1: Figure S2). The differences in the two results sets demonstrate the different characteristics of the two methods used in the analyses. For example, GFlasso is built off of an additive model, and thus will pick up on regulation signals that will not be discovered in epistatic analyses. Furthermore, GFlasso considers the whole gene-expression network to find associations between SNPs and a group of genes with highly correlated expression levels. Meanwhile, the analysis in Brem et al. [25] examined expression for each gene individually. As GFlasso tends to focus on pleiotropic 
Table 2 GO enrichment analysis of eQTL modules/hotspots found by GFlasso

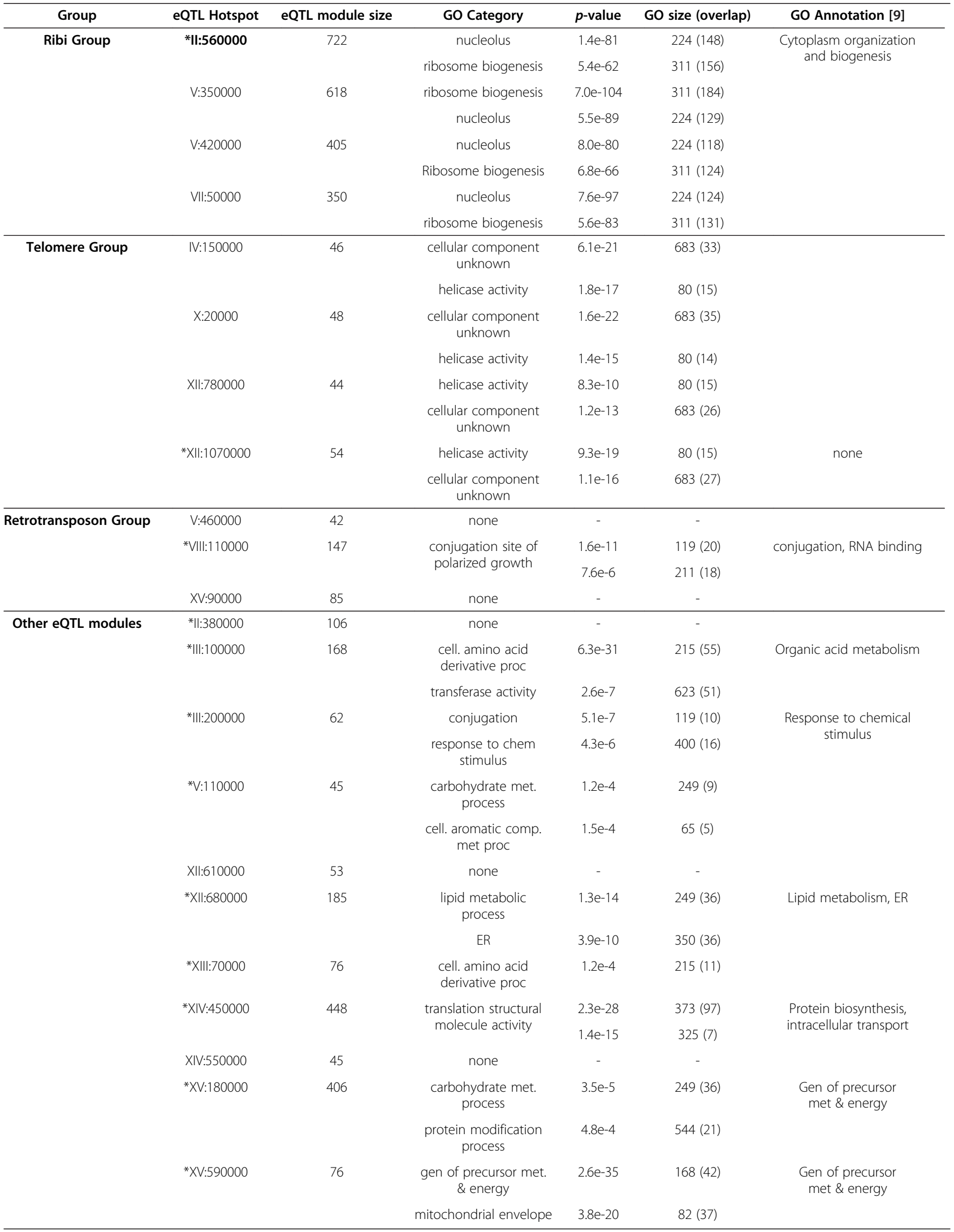




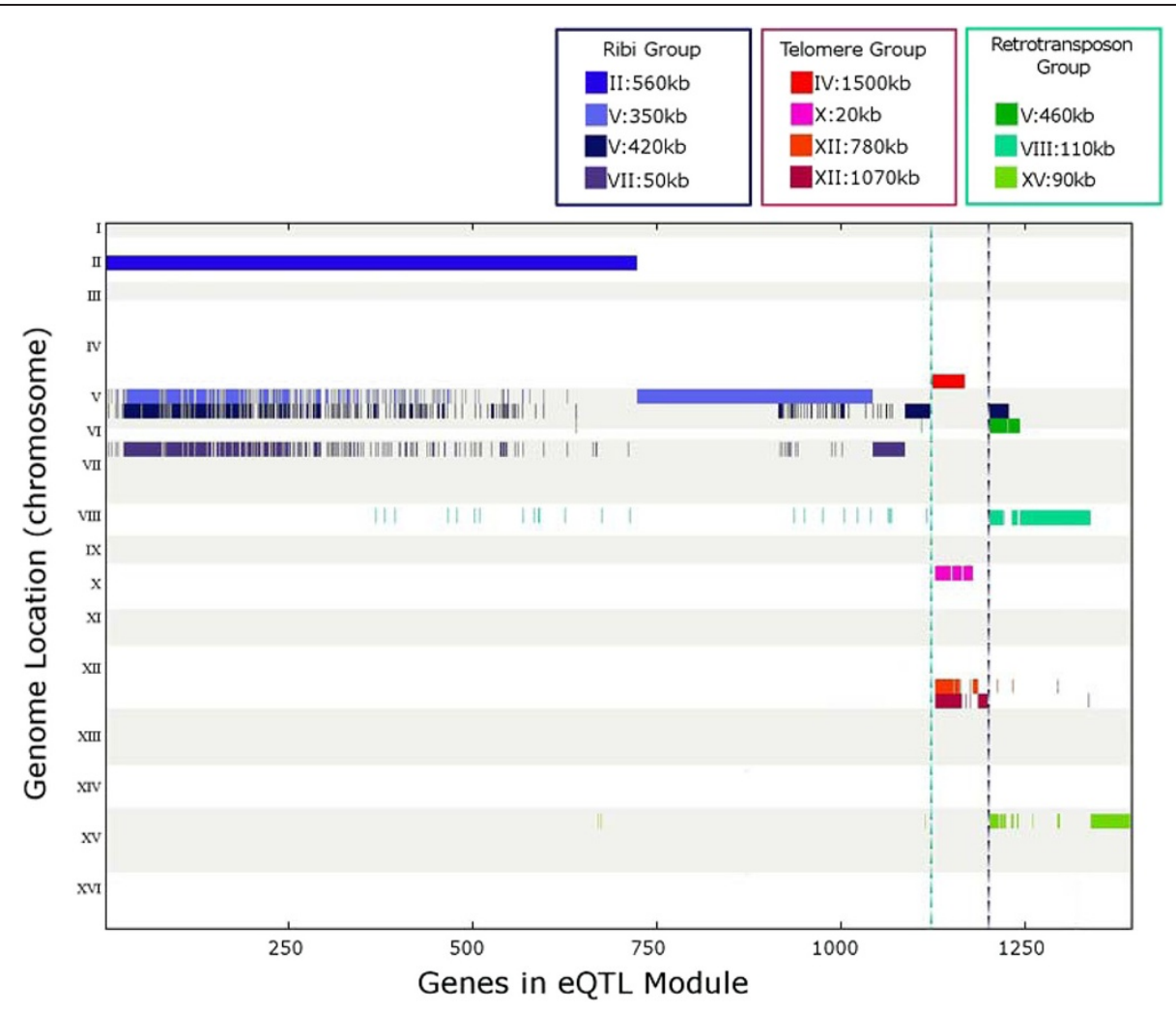

Figure 2 eQTL hotspots and their overlapping eQTL modules found by GFlasso. GFlasso found that many genes ( $x$-axis) are jointly influenced by the same genetic loci (y-axis), suggesting that these eQTL hotspots perturb an overlapping set of genes. We group these eQTL hotspots into the Ribi, telomere, and retrotransposon groups according to their overlaps in the corresponding eQTL modules. For example, the first 722 genes (plotted along the $x$-axis) all belong to the eQTL module for Il:560 kb, and many of these genes also belong to the eQTL modules derived from three other eQTL hotspots: V:350 kb, V:420 kb, and VIl:50 kb.

effects by combining information across multiple genes in the gene network, it discovered the additive effect of multiple genomic regions on gene modules. On the other hand, these signals were missed in the analysis by Brem et a.l [25], which was looking for epistatic interactions among loci with effects on individual genes.

Another recent study looked at the pleiotropic and epistatic effects among eQTL modules in this dataset [26]. In that study, Zhang et al. used a Bayesian framework to look for associations between genomic regions and modules of genes. This approach varies significantly from GFlasso in its underlying assumptions and model, especially in reference to its focus on epistatic effects. While the approaches differ, the two models are complimentary in the results that the find. For example, Zhang et al. report multiple gene modules associated with approximately 21 different genomic regions. Of these 21 genomic regions, all but two are in close proximity to the genomic regions found by GFlasso. The modules from Zhang et al.'s paper generally include fewer genes than the modules found by GFlasso and often have similar annotations. However, GFlasso finds an additional 12 genomic regions associated with an
eQTL module that are not found by Zhang et al. Three regions (V:350 kb, V:420 kb, and VII:50 kb) from the Ribi group, two regions (XII:780 kb, $1070 \mathrm{~kb}$ ) from the Telomere group, and two regions (V:460 kb, XV:90 kb) from the Retrotransposon group are found by GFlasso but not by Zhang et al. Another important difference between the methods is that while we create our eQTL modules based off of the association of multiple genes to one genomic region, Zhang et al. find multiple gene modules associated to a single genomic region. If using GFlasso, one could analyze eQTL modules using a network analysis approach to obtain a similar result.

\section{Multiple genes in three eQTL hotspots affect Ribi expression levels}

Given the evidence of multiple contributing eQTL hotspots from the GFlasso and enrichment analyses, we performed bioinformatics analysis on the three groups of eQTL hotspots: the Ribi, telomere, and retrotransposon groups. We determined the functional role that these contributing loci play on the overlapping set of genes and identified potential candidate regulators in cis to these loci. By further 


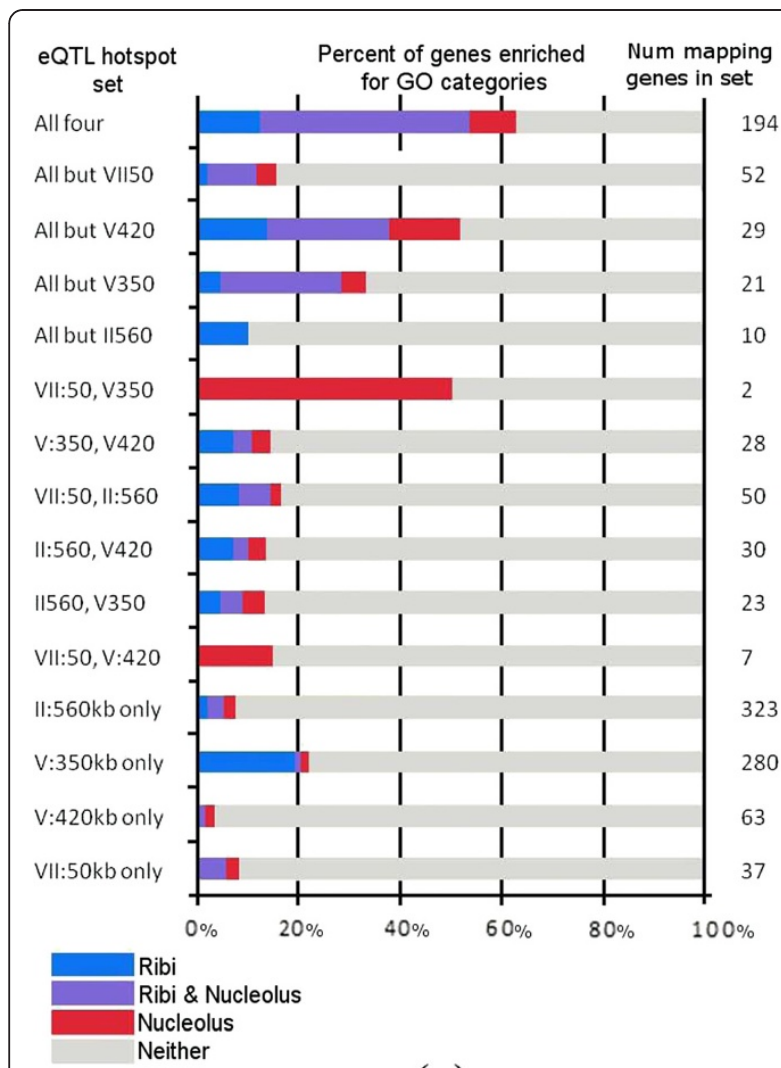

(a)

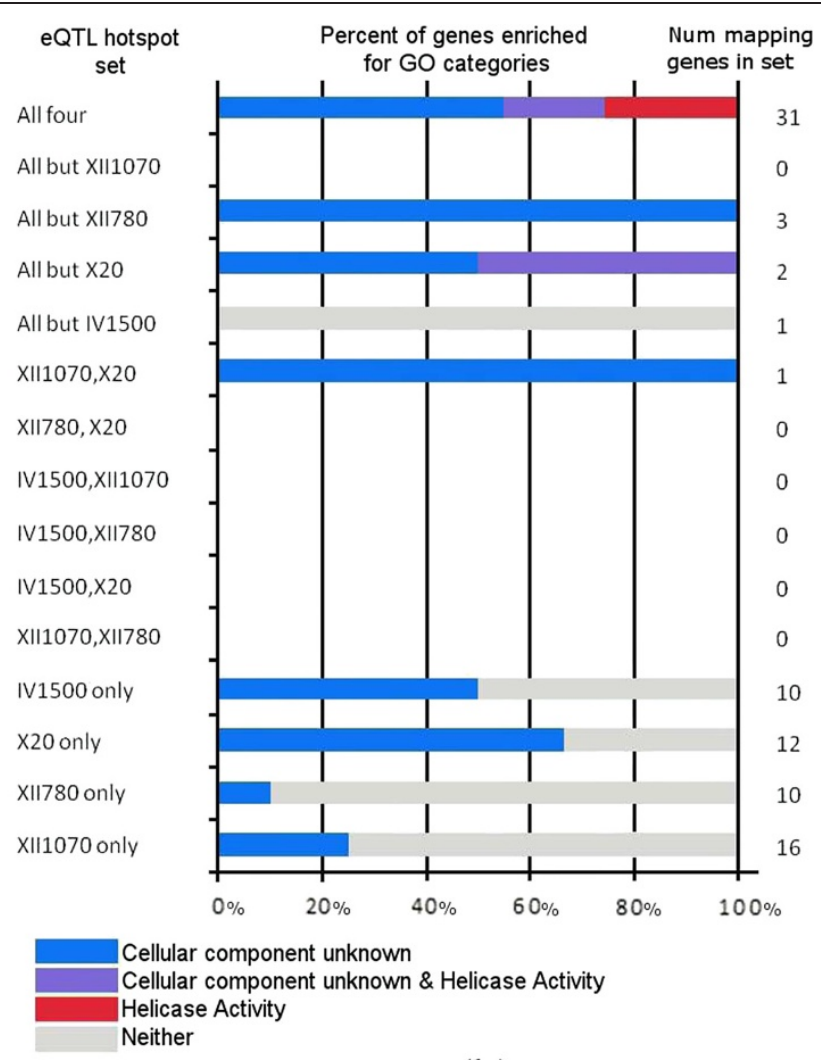

(b)

Figure $3 \mathrm{GO}$ enrichment analysis for eQTL hotspots with overlapping eQTL modules. For both the (a) Ribi group and the (b) Telomere group, we divide all of the genes in the overlapping eQTL modules into different sets (rows) based on which of the eQTL hotspots in each eQTL hotspot group they are mapped to. Genes that map to all four eQTL hotspots are placed in the set labeled "All four" in the top row. Within each of the sets, we show the percentage of genes mapping to the eQTL hotspot(s) that are annotated to one or both of the top GO enrichment categories for the eQTL hotspot group's eQTL modules along the $x$ axis with different colors. In our analysis, we focus our attention on the genes with associations to three or more eQTL hotspots in a group, as they are enriched for a common function.

examining the DNA sequence of these potential candidate regulators, we found that many of them have missense or promoter mutations between the two strains. The presence of such mutations indicates a potential change of function or expression level. In the case of the Ribi group, we further verify some of the regulators experimentally. Below, we present our analysis of the Ribi, telomere, and retrotransposon groups.

We first consider the Ribi group, which consists of four eQTL hotspots located on chromosomes II:560 kb, $\mathrm{V}: 350 \mathrm{~kb}, \mathrm{~V}: 420 \mathrm{~kb}$, and VII:50 kb. The corresponding eQTL modules overlap, with 194 genes in the overlap. 122 of these 194 genes have the GO annotation for ribosome biogenesis (GO category, type of biological function) and/ or nucleolus (GO category, type of cellular compartment).

In our analysis of these four eQTL hotspots, we found evidence of direct and indirect regulation in the Ribi regulation system and identified previously unknown potential regulators, which were confirmed through experimental validation. The creation of a Ribi protein is a multi-step process, and there are many steps along the pathway where transcriptional feedback could potentially occur. First the genes encoding the RPs (ribosomal proteins) and Ribi assembly factors must be transcribed, the transcripts translated, and then the proteins imported into the nucleus where the Ribi assembly factors assemble rRNA with the RPs into functional ribosomes. Thus, expression levels of important genes can affect the expression of genes directly, or through indirect feedback loops during any step of this process.

To limit our search for candidate regulators in cis to the four eQTL hotspots in the Ribi group, we considered genes that were located in cis to one of the four eQTL hotspots and either 1) were also found in the 194 gene overlap, implying an association to all four hotspots (8 overall, shown as green and yellow nodes in Figure 4), 2) were also known to be involved in Ribi (MAK5, UTP7, and $P B F 2$ ), or 3) were annotated as a DNA binding protein (12 overall, listed as DNA binding in Table 3). We list the candidate genes from our search in Table 3. Also, 


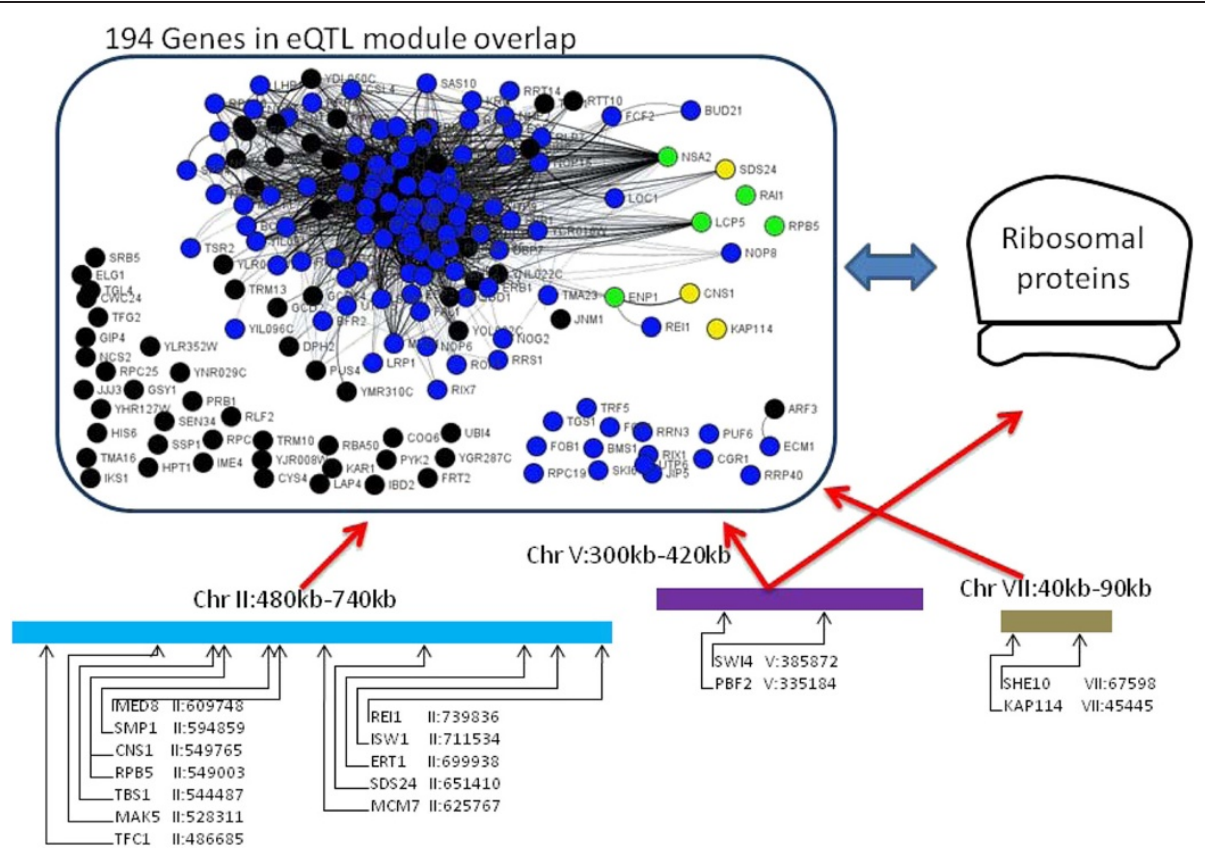

Figure 4 An illustration of gene regulation in the Ribi group of eQTL hotspots. We found four eQTL hotspots on chromosomes II, V, and VII that are all associated with the same 194 genes. 122 of the genes in this 194-gene overlap were annotated to the GO category of ribosome biogenesis (Ribi) or nucleolus (shown as blue nodes in the graph). The genes involved in Ribi are generally assembly factors that assemble rRNA and ribosomal proteins into the ribosomal unit in the nucleus. We also found an association from the V:350 kb eQTL hotspot to the ribosomal proteins. The expression levels of the ribosomal proteins are tightly coupled with the expression of the Ribi genes. Additionally, we found eight genes (shown as green and yellow nodes in the graph) in the overlap that were located in cis to one of these eQTL hotspots. The green nodes represent genes located in cis that are annotated for the Ribi or nucleolus GO categories, while the yellow nodes represent genes located in cis to one of the eQTL hotspots with a different GO annotation (see Table 3). This figure was created using GenAMap.

in Figure 4 we provide a visual overview of the regulation of these eQTL modules. Although there might be other genes in cis to these eQTL hotspots that could affect expression levels of the genes in these eQTL modules, we believe our criteria for candidate regulators led us to many of the interesting possibilities.

In both this analysis and the telomere analysis, we examine our candidate regulators by comparing the full coding and promoter sequence for the BY and RM strains. Since only 1260 unique (2956 overall) SNPs were genotyped in the eQTL dataset, these SNPs serve only as genetic markers rather than an exhaustive list of genetic polymorphisms between the two strains. Once the GFlasso analysis points us to the genomic regions or eQTL hotspot around a genetic marker, we can compare the full sequences available from public databases (see Methods) to identify missense and promoter mutations.

Validating discovered Ribi regulator genes experimentally Based on our mutation analysis, we selected five candidate regulators from three of the four eQTL hotspots for further validation using knock-out experiments ( $P B F 2$, SDS24, REI1, RAI1, and KAP114). We did not select candidates from the V:420 kb hotspot because our candidates in this region are essential genes. For each candidate regulator that we selected, we grew a knock-out strain under normal and heat shock conditions, and then compared normalized expression levels of 43 targets (from the GFlasso results) with the expression levels from the wild-type (see Methods). We consider a gene to be affected by the knock-out if its expression is more than 1.5 times greater than wild-type (or if wild-type level is more than 1.5 times greater than the knock-out). A summary of the results is available in Table 4, and a spreadsheet of the experimental results is available in the Additional file 3 . In Table 4, we report the percent of the target genes affected by the knock-out, in addition to the average and maximum fold change when compared to the wild type. These summary statistics help to demonstrate the effect that knocking out each candidate has on the targets predicted by GFlasso. We also include a pictorial representation of the results in Figure 5, showing which target genes were overexpressed in the different experiments.

Our first candidate regulator that we selected is $P B F 2$, which is located in cis to eQTL hotspot V:350 kb and is known to regulate Ribi gene expression. Ribi genes are regulated transcriptionally through the $P A C$ and $R R P E$ promoter motifs, present upstream of most Ribi genes [27]. PBF2 has previously been shown to bind to the $P A C$ motif and to regulate Ribi gene expression in 
Table 3 Candidate regulators in the Ribi group of eQTL hotspots

\begin{tabular}{|c|c|c|c|c|c|}
\hline eQTL Hotspot & Candidate & GO Category & Differentially Expressed? & Mutations & Function \\
\hline \multirow[t]{14}{*}{ II:560 kb } & TFC1 & DNA binding & No & 3 missense & RNA Pol III subunit \\
\hline & MAK5 & Ribi & No & 5 missense, 4 indel & 605 ribosome processing \\
\hline & TBS1 & DNA binding & $6.7 e-20$ & 7 missense & Unknown \\
\hline & RPB5 & Nucleolus & $9.6 e-3$ & $\begin{array}{c}\text { promoter has } 7 \text { SNPS } \\
\text { and } 1 \text { indel }\end{array}$ & RNA Poly subunit \\
\hline & CNS1 & Protein folding & $3.6 e-9$ & $\begin{array}{l}\text { promoter has } 7 \text { SNPs } \\
\text { and } 1 \text { indel, } 1 \text { missense }\end{array}$ & TPR-containing co-chaperone \\
\hline & SMP1 & DNA binding & $2.7 e-2$ & $\begin{array}{l}\text { promoter has } 7 \text { SNPs } \\
\text { and } 2 \text { indels }\end{array}$ & $\begin{array}{l}\text { Transcription factor that } \\
\text { regulates osmotic stress }\end{array}$ \\
\hline & MED8 & DNA binding & $1.2 \mathrm{e}-2$ & $\begin{array}{l}\text { Promoter has } 2 \text { SNPS, } \\
2 \text { missense }\end{array}$ & RNA Poly II mediator complex \\
\hline & MCM7 & DNA binding & No & 6 missense & DNA ATPase activity \\
\hline & SDS24 & $\begin{array}{l}\text { Molecular function } \\
\text { unknown }\end{array}$ & $7.2 \mathrm{e}-7$ & $\begin{array}{l}2 \text { missense, } 2 \text { promoter } \\
\text { SNPs }\end{array}$ & $\begin{array}{l}\text { Involved in cell separation } \\
\text { during budding }\end{array}$ \\
\hline & ERT1 & DNA binding & $2.2 \mathrm{e}-2$ & $\begin{array}{c}5 \text { missense, } 8 \text { SNPs in } \\
\text { promoter and a } 5 \\
\text { base insertion }\end{array}$ & $\begin{array}{l}\text { Transcriptional regulator of } \\
\text { nonfermentable carbon utilization }\end{array}$ \\
\hline & THI2 & DNA binding & No & None & Zinc finger protein \\
\hline & ENP1 & Ribi & No & None & $40 \mathrm{~S}$ ribosomal subunit synthesis \\
\hline & ISW1 & DNA binding & No & 1 missense & $\begin{array}{c}\text { ATPase, DNA and nucleosome } \\
\text { binding }\end{array}$ \\
\hline & REl1 & Ribi & No & 3 missense & Cytoplasmic pre-60S factor \\
\hline \multirow[t]{4}{*}{ V:350 kb } & UTP7 & Ribi & No & None & Processing of 185 rRNA \\
\hline & RAD51 & DNA binding & No & None & Strand exchange protein \\
\hline & PBF2 & Ribi & $3.0 \mathrm{e}-2$ & 9 missense & PAC binding factor \\
\hline & SWI4 & DNA binding & No & 2 missense & Transcriptional activator \\
\hline \multirow[t]{3}{*}{$\mathrm{V}: 420 \mathrm{~kb}$} & NSA2 & Ribi & $1.4 \mathrm{e}-5$ & None & $\begin{array}{c}\text { Constituent of } 605 \text { pre-ribosomal } \\
\text { particles }\end{array}$ \\
\hline & LCP5 & Ribi & $2.6 e-3$ & 1 missense & $\begin{array}{l}\text { Involved in maturation of } \\
18 \mathrm{~S} \text { rRNA }\end{array}$ \\
\hline & YER130C & DNA binding & No & 1 missense & Unknown function \\
\hline \multirow[t]{3}{*}{ VII:70 kb } & RAl1 & Ribi & No & None & Required for pre-rRNA processing \\
\hline & RTF1 & DNA binding & No & None & Subunit of RNA Pol II \\
\hline & KAP114 & Protein import into nucleus & $1.2 \mathrm{e}-8$ & 9 missense & Karyopherin \\
\hline
\end{tabular}

response to heat shock [22]. PBF2 has nine missense mutations between the BY and RM strains and is differentially expressed between the strains that have the BY allele and those that have the RM allele $(p$-value $=3.0 \mathrm{e}-2)$. These mutations suggest that $P B F 2$ may function differently in the RM and BY strain, influencing Ribi gene expression directly. In our knock-out experiment, we did find that the target gene expression levels did increase dramatically for the $\triangle P B F 2$ strain compared to the wild-type strain in response to heat shock. However, the change was negligible under normal growth conditions. Thus, our results again confirm that $P B F 2$ is involved in Ribi gene regulation.

Table 4 Summary of knock-out results for candidate regulators in the Ribi group

\begin{tabular}{|c|c|c|c|c|c|c|c|c|c|c|}
\hline & $\triangle S D S 24$ & $\triangle P B F 2$ & $\triangle R E I 1$ & $\triangle R A / 1$ & $\triangle K A P 114$ & $\triangle S D S 24 \mathrm{HS}$ & $\triangle P B F 2 \mathrm{HS}$ & $\triangle R E I 1 \mathrm{HS}$ & $\triangle R A / 1 \mathrm{HS}$ & $\triangle K A P 114 \mathrm{HS}$ \\
\hline$\%$ targets affected & $4.7 \%$ & $4.7 \%$ & $74.4 \%$ & $83.7 \%$ & $4.7 \%$ & $7.0 \%$ & $79.1 \%$ & $79.1 \%$ & $86.0 \%$ & $60.5 \%$ \\
\hline max change & 1.691 & 1.983 & 2.686 & 2.934 & 1.838 & 1.531 & 5.117 & 5.700 & 5.977 & 3.847 \\
\hline avg. change & 1.168 & 1.245 & 1.641 & 1.824 & 0.962 & 1.174 & 2.206 & 2.241 & 2.417 & 1.641 \\
\hline \# controls affected & 0 & 0 & 3 & 2 & 1 & 0 & 1 & 5 & 3 & 1 \\
\hline$p$-value & 1 & 1 & $8.6 e-6$ & $2.3 e-8$ & 1 & 0.55 & $1.3 e-8$ & $6.1 e-5$ & $7.6 e-8$ & $2.6 e-5$ \\
\hline
\end{tabular}

Genes with expression levels greater than 1.5 times the wild type, or less than $2 / 3$ the wild type, levels are considered to be affected by the knockout. The max change and average change rows represent the fold change in the expression level compared to the wild time. HS denotes that the yeast were heat shocked before extracting RNA. There were 20 total control genes. The p-value represents a Fisher's exact score between the genes affected in the target and control groups. 


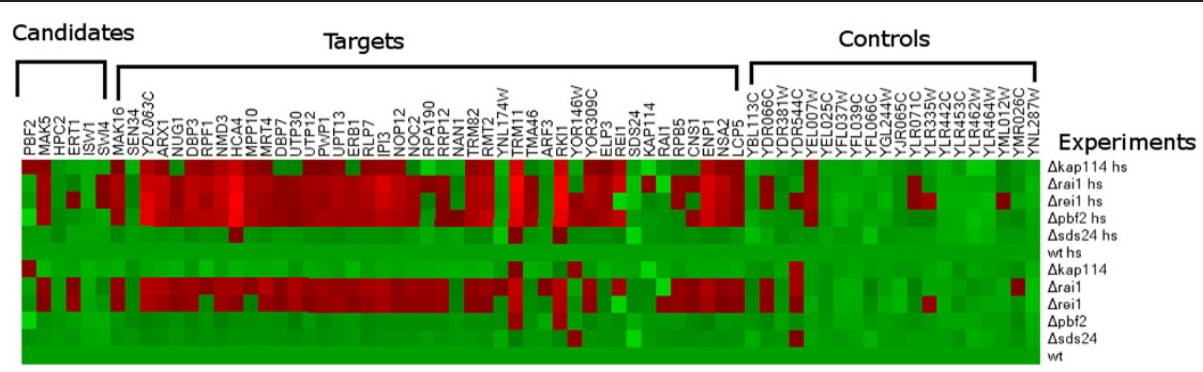

Figure $\mathbf{5}$ Knockout experiment results. We show the results from the knockout experiments using a graphical representation of the data. This plot was generated with Heatmap Builder [48] using the log values of the normalized gene expression measurements. The red pixels in the graph represent an over-expression of the genes in the experiment, while the bright green pixels represent under-expression. These results suggest that GFlasso correctly identified target genes that change expression due to mutations in the eQTL hotspots that were identified.

We considered two candidate regulators from the VII:50 kb eQTL hotspot: KAP114 and RAI1. KAP114 is a nuclear importer that could have an indirect effect on Ribi gene transcription. KAP114 is one of the 194 genes in all four eQTL modules. Nuclear import is an important step in Ribi; the Ribi proteins are translated in the cytoplasm and then KAP proteins import them into the nucleus, where these proteins assemble the RPs and rRNA into ribosomes [28]. Although KAP114 has not yet been implicated in Ribi protein import, Ribi proteins and RPs account for most of the incoming nuclear traffic in a cell [28]. Therefore, if transport of these proteins into the nucleus was affected, the rate of Ribi transcription could be affected through a feedback loop. RAI1 is a known Ribi gene; based on our results we hypothesize that RAI1 affects the expression of other Ribi genes through a feedback loop or an unknown regulatory role. Our knock-out experiments found that predicted target genes were affected by the $\triangle R A I 1$ strain in normal and in heat shock conditions. This is an interesting result as RAI1 has no known regulatory role in Ribi gene expression. We found that the $\triangle K A P 114$ strain had higher expression levels of many of the target genes $(60 \%)$ during a heat shock, but that the expression of the targets was not affected under normal conditions. These results support the discovery of the V:50 kb hotspot as an important genomic region involved in the transcriptional regulation of Ribi genes.

GFlasso found associations from many SNPs on chromosome II to the Ribi genes. Thus, due to the large size of the eQTL hotspot on chromosome II:560 kb (about $200 \mathrm{~kb}$ ), we had many candidate regulators to consider. Here we report an interesting mutation in the promoter region between $C N S 1$ and RPB5. CNS1 and RPB5 are both associated to all four eQTL hotspots in the Ribi group. There are seven SNPs and an indel in the promoter region, which could potentially affect the expression of both genes. RPB5 is a component of RNA polymerase; a change in expression levels would directly perturb Ribi gene levels. Also, previous computational studies list CNS1 as a possible Ribi regulator, although its involvement is unclear $[8,9]$. We also considered two other candidate regulators that we found: SDS24 and REI1. SDS24 is located near II:651 kb and REI1 is located near II:739 kb; both locations are in the eQTL hotspot and are part of the eQTL module. In this experiment, we created knockout strains for SDS24 and REI1, as CNS1 and RPB5 are essential genes. We did not find a significant difference in the target gene expression in the $\triangle S D S 24$ strain. We did find, however, that the Ribi target genes were over-expressed in the $\triangle R E I 1$ knockout strain when compared to the wild type. This is another interesting observation, as REI1 is a Ribi gene with no known regulatory role.

In summary, we have looked at three eQTL hotspots belonging to the Ribi group of hotspots and have shown experimentally that candidate regulators in each eQTL hotspot affect the expression of the target genes predicted by GFlasso. One interesting observation is that Ribi genes with no known regulatory role, such as REI1 and RAI1, affected the expression of other Ribi genes (targets). This suggests that these Ribi genes might have an unknown regulatory role, or the association may uncover a feedback loop where other genes are over-expressed to compensate for the loss of other Ribi genes. On another note, out of the 43 targets predicted by GFlasso, only four genes were not affected by the regulators we tested, suggesting that GFlasso predicts the targets with high accuracy. Finally, it is interesting to note that all four validated regulators have a stronger effect in the case of heat shock, demonstrating the inconsistent effect that mutations can have in different growth environments.

\section{eQTL hotspots harbor mutations in NUP2, RIF2, and SIR3 that potentially affect telomere silencing}

We now consider the four eQTL hotspots in the telomere group: IV:1500 kb, X:20 kb, XII:780 kb, and XII:1070 kb. Each of these eQTL hotspot are associated with the same 31 genes, and six additional genes are associated with three of the four eQTL hotspots. All 37 genes lie in telomere regions and three (IV:1500 kb, 
$\mathrm{X}: 20 \mathrm{~kb}$, and XII:1070 kb) of the four eQTL hotspots also lie in telomere regions. This suggests that there is coordinated or additive regulation among these four eQTL hotspots to turn on the expression of telomere genes. GO enrichment analysis found that these genes are enriched for the GO functional annotation "helicase activity" ( $p$-value $=2 \mathrm{e}-17$ ) and the GO component annotation "cellular component unknown" ( $p$-value $=2 \mathrm{e}-17)$. This suggests that these genes share common function and interact with DNA as a helicase. Because many of the genes do not have a cellular component annotation, they might be understudied or not ordinarily expressed.

We considered the known function of each gene in the set individually and found seven yeast $Y R F 1$ genes (YRF1-1, YRF1-2, YRF1-3, YRF1-4, YRF1-5, YRF1-6, and YRF1-7), see Table 5. We also found that the other 30 genes were all either "proteins of unknown function," or "helicase-like proteins encoded within the telomeric $\mathrm{Y}^{\prime}$ element" (SGD database). Because the annotations were common across the genes in the set, we performed a sequence BLAST against YRF1-1 and found that 36 of the 37 genes had high homology to the YRF1-1 transcript (BLAST eValue < 1e-36). The 29 non-YRF1 genes had no known functionality, despite their homology to the YRF1 genes. We conclude that these genes are copies of YRF1 in the yeast telomeres. The homology also suggests that these genes cross-hybridize to each other's probes on the microarray; if any one of the genes regulated by these four eQTL hotspots is expressed, all of the genes would appear to be expressed on the microarray. The homology of this module has been previously observed [9], however, in the RM wild type strain, the YRF1 genes have a significantly lower expression level than in the BY mutant strain $(t$-test $p$-value $=1.1789 \mathrm{e}-26)$, which cannot be explained by the hypothesis of cross-hybridization. It appears that there is some kind of regulation turning on, or failing to turn off, at least one of the YRF1 genes in the BY strain.

In order to explain the difference in $Y R F 1$ and $Y R F 1$-like gene expression between the RM and BY strains, we considered what is known about the YRF1 genes. The YRF1 genes are known to be a backup plan for telomerase. Telomerase is the protein complex essential for maintaining telomere length [29]. The loss of telomerase results in the gradual shortening of the telomeres and in eventual cell arrest, unless the telomeres are lengthened through the YRF1 pathway [30]. There are many copies of YRF1 located in yeast telomeres [31]. YRF1 genes are not expressed in wild type cells, probably due to telomere silencing. However, it appears that as the telomeres shorten, silencing information is removed, leading to the expression of the YRF1 genes [31]. The YRF1 genes contain several helicase motifs and are believed to extend the telomeres through DNA homologous recombination, largely because other helicases participate in homologous recombination and genes important in homologous recombination are essential for survival without the proper function of telomerase [31].

Therefore, one possibility to explain the expression of YRF1 is impaired telomerase function. Telomerase is made up of five proteins, two of which are functionally essential: TLC1 and EST2 [31]. EST2, a reverse transcriptase, is located in cis to the eQTL hotspot at XII:780 kb in the telomere group. We considered the sequence of the RM11-1a strain against the S288c strain and found five missense mutations in the EST2 transcript (EST2 was not differentially expressed between the two strains, $p$-value $>.2$ ), including an $\mathrm{R}$ to $\mathrm{Q}$ mutation in the reverse transcriptase domain. These mutations suggest that EST2 could be impaired in its function, allowing for the shortening of telomeres and the activation of the YRF1 genes. However, we conclude that the loss of EST2 function is unlikely, given the popularity of the S288c strain and its use as a "normal" control for functional telomerase in yeast telomere studies [30]. We therefore suggest other pathways that potentially regulate YRF1 gene expression.

Another possibility to explain the YRF1 gene expression is the loss of telomere silencing genes. NUP2 (XII:780 kb) and RIF2/SIR3 (XII:1070 kb), are telomere silencing genes cis to hotspots in the telomere group. Dilworth et al. [32] report that NUP2 (part of the nuclear pore complex) localizes in the nucleus with yeast telomeres; ChIP-chip experiments reveal that NUP2 has a telomere binding preference. These results, combined with the association found by the GFlasso, suggest that NUP2 (seven missense mutations) is a player in telomere silencing. In regards to RIF2, RAP1 is also known to be involved in telomere silencing [33], and in this role it is

Table 5 Candidate regulators in the telomere group of eQTL hotspots

\begin{tabular}{|c|c|c|c|c|}
\hline eQTL Hotspot & Candidate & Differentially Expressed? & Mutations & Function \\
\hline IV:1500 kb & YRF1-1 & $6.4 \mathrm{e}-6$ & 5 base insertion $186 \mathrm{nt}$ upstream & YRF1 gene \\
\hline $\mathrm{X}: 20 \mathrm{~kb}$ & YJL225C & $1.1 \mathrm{e}-4$ & 10 base insertion $237 n t$ upstream & YRF1-like gene \\
\hline \multirow[t]{2}{*}{ XII:780 kb } & EST2 & No & 5 missense & Telomerase component, reverse transcriptase \\
\hline & NUP2 & No & 7 missense & Nuclear pore protein involved in telomere silencing \\
\hline \multirow[t]{2}{*}{ XII:1070 kb } & SIR3 & $5.3 e-3$ & 12 missense & Involved in telomere silencing \\
\hline & RIF2 & No & 6 missense & Involved in telomere silencing \\
\hline
\end{tabular}


assisted by RIF2; deletions in RIF2 affect telomere length [34]. SIR3 is also recruited to the telomere regions by $R A P 1$, and cells lacking telomerase have increased concentration levels of the SIR3 protein [30]. SIR3 (twelve missense mutations) and RIF2 (six missense mutations) are both located in cis to the XII:1070 kb eQTL hotspot. Previous computational analyses of this dataset have similarly implicated RIF2 as a possible regulator of telomere genes [35].

A final possibility to explain the $Y R F 1$ gene expression is the loss of silencing sequence in the DNA, leading to the expression of a YRF1 transcript. YRF1-1 (IV:1500 kb) and YJL225C (X:20 kb) both have mutations that could have this effect. YRF1-1 has a five-base indel located 186 bases upstream in its promoter region, and YJL225C has a ten-base indel located 237 bases upstream in its promoter region. These mutations could potentially remove silencing information for these genes; the genotype at the eQTL hotspot is indicative of the expression level in both cases ( $p$-value $=1 \mathrm{e}-4$ and $1 \mathrm{e}-6$ respectively).

In conclusion, we investigated three possibilities where mutations in the BY strain could lead to the expression of at least one YRF1 gene transcript. We suggest that telomerase is not impaired in the BY strain, however, mutations in telomere silencing genes and in promoter regions of YRF1 genes are likely candidates that may work together or in parallel to either turn on or silence $Y R F 1$ gene expression.

\section{GFlasso uncovers 17 retrotransposon insertions}

The retrotransposon group of eQTL hotspots are located on V:460 kb, VIII:110 kb, and XV:90 kb. The eQTL modules for each of these eQTL hotspots differ in size $(42,85$, and 147 genes), although they all influence a common set of 20 genes, with 35 genes associated with two of the three eQTL hotspots. Although the VIII:110 kb eQTL module is enriched for conjugation (due to its close proximity with the mutated GPA1 gene [17]), neither of the other two eQTL modules are enriched for a GO category. From our analysis, we found that the non-overlapping genes in these eQTL modules are not related to the genes involved in the overlap of the eQTL modules.

When we considered each of the 35 genes, we found that 15 of the genes are highly homologous (BLAST score of less than 1e-200 when queried against each other) and are annotated as Ty retrotransposons in the SGD database. We investigated the significance of finding 15 retrotransposons in the same eQTL module. A computational study [36] identified 331 retrotransposons in the yeast genome, 94 of which correspond to retrotransposon genes listed in the SGD. Of these 94 genes, 21 are included in the Brem and Kruglyak [13] dataset; it is unlikely that 15 of these 21 genes would end up in the same eQTL module of size 35 ( $p$-value $=1.27 \mathrm{e}-30)$.
In yeast there are five types of retrotransposons, referred to as the Ty genes: Ty1, Ty2, Ty3, Ty4, and Ty5, with Ty1 being the most frequent in the genome [36]. Retrotransposons are scattered throughout the genomes of eukaryotes and function like a virus that is transcribed into an mRNA intermediate. This mRNA intermediate, through a reverse transcriptase, is then inserted back into the genome as cDNA, playing an important role in genome evolution [36,37]. It is estimated that Ty retrotransposon mRNA accounts for about $1 \%$ of the total mRNA in a cell. However an insertion into the chromosomal DNA only happens between $10^{-7}$ and $10^{-8}$ times per cell division cycle, suggesting that the insertion of the Ty genes is regulated post-transcriptionally $[38,39]$. The transcriptional regulation of the Ty genes happens through the TATA-box and other information in the promoter, and has been linked to the suppressor of transposition (SPT) genes and the STE genes [38].

Due to the sequence homology of these genes, it is probable that the observed co-expression is a result of cross-hybridization. However, we were interested to find genes in the three associated eQTL hotspots that could account for the transcriptional diversity between the strains. We found a few candidate STP and STE genes based solely on location, SPT15 at V:464 kb and STE2O at VIII:94 kb. However, sequence analysis revealed that SPT15 is perfectly conserved between the RM and the BY strain. STE2O had eight missense mutations and a few SNPs in its promoter region.

Interestingly, we found a retrotransposon located in cis to each of the three eQTL hotspots; each homologous to the 15 retrotransposons discovered in the eQTL module overlap. These three retrotransposons, YER138C (V:449 kb), YHLOO9W-B (VIII:85 kb), and YOL104W-A (XV:118 kb) are present in the S288c strain, but not in the RM strain sequence. This could be due to errors in the assembly of the RM sequence, but it likely that the insertion happened after these two strains diverged. We additionally considered each of the 15 Ty1 genes in the eQTL module overlap among these three contributing eQTL hotspots and found that only one Ty1 gene was present in both strains. Thus, we have found 17 total (14 in the dataset and 3 in cis to eQTL hotspots) retrotransposon insertions between the BY and RM strain, leaving open the possibility for other insertions as well. Additionally, among the 20 genes in the overlapping set of 35 that were not retrotransposons, we found that 13 of them were within $10 \mathrm{~kb}$ of a retrotransposon site and therefore could be expressed differently between the two strains because of the retrotransposon.

GFlasso has therefore uncovered a case where retrotransposon insertions have occurred since the BY and RM strain diverged. The occurrence of such retrotransposon insertion events in separate populations is not surprising and can be found by the direct comparison of 
the genome sequences of the two yeast strains. However, our analysis shows that in the absence of the full genome sequence information, GFlasso has the potential to discover systematic sequence differences such as gene insertions by investigating their impact on the expression levels solely based on an eQTL dataset.

\section{Discussion and conclusions}

Many of the previous methods for discovering eQTLs from genotype and gene-expression datasets have been concerned with testing the hypothesis of association between an individual genotype and the expression of each gene [1]. However, there is a great deal of evidence that the elements in the genome and transcriptome interact with each other in performing a biological function; it has been widely recognized that the computational methods for detecting eQTLs should take into account this complex interaction pattern. Structured association mapping methods are powerful computational methods that directly map the quantitative-trait (gene-expression) network to genotypes, explicitly combining information across multiple correlated traits to increase the power of detecting association. There are a variety of structured association mapping methods available including others that consider gene networks, such as TreeLasso [40], in addition to other methods that consider population structure [41] or known information about the genome [42]. Indeed, it is important to take into account the inherent structures present in the data when looking for associations. Not only are there powerful methods available, but these methods are also available in a unified visualization software framework through GenAMap [16,43], that is easily assessable by analysts and experimentalists.

In this study, we re-analyzed the eQTL dataset [13] from the genetic cross of two yeast strains (BY and RM) using a structured association mapping method called GFlasso and discussed the new insights into yeast gene regulation that were provided by our analysis. The yeast eQTL dataset provides an excellent test-bed for comparing various computational methods, as it has been extensively analyzed. We showed that GFlasso, coupled with additional bioinformatics analysis, led to significant biological findings that had not been discovered by other methods, and we demonstrated the potential of GFlasso for future analyses of eQTL datasets that are becoming available for various organisms, tissue types, and diseases

While the pleiotropic control of multiple genes by a genetic locus, called an eQTL hotspot, has been previously reported in analyses of many different eQTL datasets, our analysis of the yeast eQTL dataset revealed another layer of complexity in gene regulation by uncovering the pleiotropic effect of multiple genetic loci on multiple genes. The literature has yet to report this type of pleiotropic effects of multiple contributing genetic loci. Although our analysis in this study was focused on yeast, we suspect that the pleiotropic regulation of genes by multiple contributing eQTL hotspots is commonplace in many other eQTL datasets. Our results show that it may be worthwhile to revisit eQTL datasets with this new perspective, especially as more powerful computational methods become available. Furthermore, our results demonstrate the advantages of using structured association mapping in future studies to uncover weak signals and also of considering multiple genomic regions when identifying regulatory genes.

Our close investigation of the three groups of eQTL hotspots that control an overlapping set of genes led to new insights into Ribi gene regulation, telomere silencing, and retrotransposon activity and suggested potential regulators. By identifying missense and promoter mutations in the full DNA sequence of the candidate regulators, in addition to validation knock-out experiments, we provided strong evidence that these candidate regulators influence the gene expression levels of many genes in these biological pathways. In addition, we showed that prior studies of these individual genes in the literature support many of the hypotheses that the candidate regulators have the functional role suggested by our analysis. As yeast is one of the model organisms that have been studied extensively, a plethora of information is already available. This information includes the full genome sequence as well as detailed investigations of many of the genes; we were able to confirm the results of our analysis by comparing the results with this information. For many complex diseases, in other organisms where the same kind of extensive knowledge base is not yet available, we expect structured association mapping to serve as a powerful computational tool for new discoveries.

Our bioinformatics analysis of the three groups of eQTL hotspots opens up many research questions on the regulation of Ribi genes and telomere silencing that need to be further investigated in follow-up studies. Although our analysis suggests that the candidate regulators on different eQTL hotspots affect the same set of genes in a coordinated manner, understanding the exact mechanism of such coordination would require further research. For example, NUP2 and SIR3 on two different loci in the telomere group of eQTL hotspots have been found to regulate telomere silencing in both this analysis and previous studies of these genes. However, exactly how this interaction between these two genes occurs remains unexplored. This novel interaction could lead to further insight into how NUP2 is involved in telomere silencing and perhaps uncover further interactions between various genetic loci that turn genes on and off.

Finally, GFlasso is designed to identify additive effects of multiple genetic loci on correlated traits, and thus, 
the effects of multiple eQTL hotspots on each gene were additive. An interesting future research direction would be to consider epistatic interactions among multiple eQTL hotspots, where the effect of a given eQTL hotspot is not independent of the genotypes of other eQTL hotspots. As detecting epistatic effects on individual genes is widely known as a computationally intensive task, the more challenging problem of detecting epistatic effects on multiple genes with pleiotropic effects would require a significant advance in computational tools.

\section{Methods}

\section{Creating a network from gene expression data}

GFlasso [7] takes a gene-interaction network as input, along with the genotype and gene-expression data, and performs a correlated association analysis to identify genomic regions that perturb correlated traits in the network. In order to obtain a gene-interaction network to use as an input to GFlasso, we used the algorithm for learning a topological overlap matrix as described in Zhang \& Horvath [14] that was applied to the same dataset in Zhu et al. [9]. The resulting network has the properties of being modular and scale free with a few hub genes having high connectivity and controlling many other genes. Genes that appear correlated in this network often share common functionality and therefore are likely to be regulated by the same regions of the genome [9]. Complete details of our network construction method are available in Additional file 1: Supplementary Methods.

In order to improve the computational efficiency of running GFlasso, instead of performing a single GFlasso analysis on the full network of 5637 genes, we divided the full network into a set of smaller subnetworks, ran GFlasso on each subnetwork in parallel, and combined the results. We divided the full network into subnetworks so that the main connectivity structure with hub nodes and strong edges in the original network is preserved, while edges for weak correlation are ignored. We first identified connected components (four of size 16, 29,45 , and 3429) in the full network, where there are no edges going across different subnetworks. For the large connected component of size 3429, we ran a graphclustering method called spectral clustering [44] to further divide it into eight smaller subnetworks. Complete details are provided in the Additional file 1: Supplementary Methods.

\section{GFlasso analysis}

GFlasso is a sparse multivariate regression method that we have previously developed for finding a correlated genome association for multiple related traits given a trait network and genotype/phenotype dataset [7]. GFlasso extends the standard lasso [45] that uses an $L_{1}$ penalization to shrink the regression coefficients (or parameters for association strengths) towards zero and obtain a sparse estimate with many zero-valued coefficients for SNPs with no associations. The lasso has been widely used for single-trait association analysis. In addition to the lasso penalty, GFlasso introduces another penalty function, called a graph-guided fused-lasso penalty, which is constructed from the trait network and plays the role of enforcing a soft constraint that highly correlated traits in the network are influenced by a common region in the genome. Given an $N \mathrm{x} J$ genotype data matrix $X$, where $N$ is the number of strains and $J$ is the number of SNPs, and an $N \times K$ gene-expression data matrix $Y$, where $K$ is the number of genes, GFlasso estimates the regression coefficients $B$, a $J \times K$ matrix, for association strengths by solving the following optimization problem:

$$
\begin{aligned}
B=\operatorname{argmin} & \sum_{k}\left(y_{k}-X \beta_{k}\right)^{T}\left(y_{k}-X \beta_{k}\right)+\lambda \sum_{k} \sum_{j}\left|\beta_{j k}\right|+\gamma \\
& \times \sum_{(m, l) \in E} f\left(r_{m l}\right) \sum_{j}\left|\beta_{j m}-\operatorname{sign}\left(r_{m l}\right) \beta_{j l}\right|
\end{aligned}
$$

where $y_{k}$ and $\beta_{k}$ denote the $k$ th column of $Y$ and $B$. The last term in the above equation is the graph-guided fusedlasso penalty that encourages two association strengths $\beta_{j m}$ and $\beta_{j l}$ to have similar values if the $m$ th and $l$ th traits are correlated and connected with an edge $(m, l) \in E$.

The $\lambda$ and $\gamma$ in the above equation are the regularization parameters that control the amount of penalization. We found the optimal values for $\lambda$ and $\gamma$ through crossvalidation as follows. We divided the full dataset into one training set (104 strains) and one validation set (10 strains), ran GFlasso on the training set for different values of $\lambda$ and $\gamma$, and computed prediction error on the validation set. This was done on one split of the data only due to the computation running time of the algorithm. We selected the values of $\lambda$ and $\gamma$ with the lowest validation-set error as the optimal values. Instead of performing a grid search over $\lambda$ and $\gamma$, we first fixed $\gamma=0$ and searched for an optimal value of $\lambda$ on the $\log 10$ scale. The $\lambda$ with the smallest prediction error is then used to search for an optimal value of $\gamma$ on the $\log 10$ scale. Then, we ran a finetuned search for $\lambda$ given $\gamma$. Once we obtained the optimal estimate of association strengths $B$, we considered all $\mathrm{SNP} /$ gene pairs corresponding to non-zero entries in $B$ as significantly associated.

\section{GenAMap}

We developed a software platform called GenAMap $[15,16]$ that implements the full analysis pipeline of structured genome-phenome association analysis and provides tools to visualize the results from the analysis. The GFlasso analysis pipeline that we used in this paper is fully integrated into GenAMap, including creating the trait network and running the GFlasso program on the 
eQTL data. GenAMap presents the user with interactive visualizations that show the structure of the genome and the phenome when browsing association results. GenAMap is a visual analytic tool for a variety of other spare-structured regression techniques for structured genome-phenome associations [40,41]. GenAMap also helps to scale the computational running time of these algorithms (running time of various algorithms, including GFlasso is discussed in our previous work $[7,43]$ ).

\section{Identifying mutations between the strains}

In order to identify the genotypic differences in genes across two strains, we downloaded the RM11-1a sequence for each protein of interest from the Saccharomyces cerevisiae RM11-1a sequencing project website [46] and used it as the query for BLASTp search [47], limiting the results to the Saccharomyces cerevisiae S288c strain. The full protein sequences that we obtained as results for each query were used to identify mutations between the two strains. In the cases of promoter mutations, we took the 500 bases upstream of the gene in the RM sequence and performed a BLASTn search [47] in the BY strain.

\section{Preparing knock-out yeast strains}

Knockout strains of yeast were obtained from Open Biosystems. Overnight cultures of yeast grown in YEPD medium at $25^{\circ} \mathrm{C}$ were diluted ten-fold into $25 \mathrm{ml}$ of YEPD and grown at $25^{\circ} \mathrm{C}$ to $5 \times 10^{8}$ cells $/ \mathrm{ml}$. Then, $12.5 \mathrm{ml}$ of the culture was grown for another $15 \mathrm{~min}$ at $25^{\circ} \mathrm{C}$, and $12.5 \mathrm{ml}$ was shifted to $38^{\circ} \mathrm{C}$ for $15 \mathrm{~min}$. Cells were harvested by centrifugation, washed once in sterile water, and cell pellets were frozen.

\section{Measuring the expression levels}

To measure the expression levels of the selected genes in the wildtype and the five knockout mutant strains, we performed nanoString expression analysis. Total RNA was extracted using the Qiagen RNeasy Plant kit (Cat \#74904). $800 \mathrm{ng}$ of total RNA was mixed with the nanoString probe set and incubated at $65^{\circ} \mathrm{C}$ overnight (12-18 hours). The reaction mix was then loaded on the nanoString nCounter Prep Station for binding and washing, using the default program. The resultant cartridge was then transferred to the nanoString nCounter digital analyzer for scanning and data collection. A total of 600 fields were captured per sample. The raw data, in a form of digital counts for each of the probe target genes in every sample, were first adjusted for binding efficiency and background subtraction using the manufacturer included positive and negative controls, following nCounter data analysis guidelines. Second, mutant strain data sets were normalized to the control wildtype strain using the 20 included control genes. The normalized data sets were used to determine if the expression level of a gene in a mutant was different from that in the wild-type control. Results were visualized using Heatmap builder [48].

\section{Additional files}

Additional file 1: Supplementary Methods.

Additional file 2: Supplementary GFlasso results.

Additional file 3: Supplementary knockout experiment results.

Competing interests

REC and EPX have filed a patent application for GenAMap, the software used to conduct this analysis.

\section{Authors' contribution}

REC carried out the computational analysis and drafted the manuscript. SK provided key insight throughout the analysis and drafted the manuscript. JLW provided expert advice and helped coordinate the molecular experiments. WX performed the nanostring experiments. EPX directed the project and drafted the manuscript. All authors read and approved the final manuscript.

\section{Acknowledgements}

We would like to thank Minli Xu for his help to implement the epistatis analysis for our comparison results and Joshua Woodward for his help to run the nanostring experiments. This work was supported by the Defense Advanced Research Projects Agency [Z931302]; National Science Foundation [DBI-0640543, IIS-0713379]; National Institutes of Health [1R01GM087694, 1R01GM093156, 0015699]; and an Alfred P. Sloan Research Fellowship to EPX.

\section{Author details}

${ }^{1}$ Joint Carnegie Mellon - University of Pittsburgh PhD Program in Computational Biology, Carnegie Mellon University, Pittsburgh, PA, USA. ${ }^{2}$ Lane Center for Computational Biology, Carnegie Mellon University, Pittsburgh, PA, USA. ${ }^{3}$ Department of Biological Sciences, Carnegie Mellon University, Pittsburgh, PA, USA. ${ }^{4}$ Machine Learning Department, Carnegie Mellon University, Pittsburgh, PA, USA.

Received: 9 November 2012 Accepted: 12 March 2013 Published: 21 March 2013

\section{References}

1. Gilad Y, Rifkin SA, Pritchard JK: Revealing the architecture of gene regulation: the promise of eQTL studies. Trends Genet 2008, 24(8):408-445.

2. Brem RB, Yvert $G$, Clinton R, Kruglyak L: Genetic dissection of transcriptional regulation in budding yeast. Science 2002, 296(5568):752-755.

3. Chen Y, Zhu J, Lum PY, Yang X, Pinto S, et al: Variations in DNA elucidate molecular networks that cause disease. Nature 2008, 452:429-435.

4. Stranger BE, Nica AC, Forrest MS, Dimas A, Bird CP, et al: Population genomics of human gene expression. Nat Genet 2007, 39:1217-1224.

5. West MAL, Kim K, Kliebenstein DJ, van Leeuwen H, Michelmore RW, et al: Global eQTL mapping reveals the complex genetic architecture of transcript-level variation in Arabidopsis. Genetics 2007, 175(3):1441-1450.

6. Yaguchi $\mathrm{H}$, Togawa $\mathrm{K}$, Moritani M, Itakura M: Identification of candidate genes in the type 2 diabetes modifier locus using expression QTL. Genomics 2005, 85(5):591-599.

7. Kim S, Xing EP: Statistical estimation of correlated genome associations to a quantitative trait network. PLoS Genet 2009, 5(8):e1000587.

8. Lee SI, Dudley AM, Drubin D, Silver PA, Krogan NJ, et al: Learning a prior on regulatory potential from eQTL data. PLoS Genet 2009, 5(1):e1000358.

9. Zhu J, Zhang B, Smith EN, Drees B, Brem RB, et al: Integrating large-scale functional genomic data to dissect the complexity of yeast regulatory networks. Nat Genet 2008, 40(7):854-861.

10. Leonardo B, Enrico P, Stefan B, François C, StuartA C, et al: Bayesian Detection of Expression Quantitative Trait Loci Hot Spots. Genetics 2011, 189(4):1449-1459.

11. Lei B, Xuefeng X, Yan C: Expression QTL Modules as Functional Components Underlying Higher-Order Phenotypes. PLoS One 2010, 5(12):e14313. 
12. Wei Z, Jun Z, Schadt EE, Liu JS: A Bayesian Partition Method for Detecting Pleiotropic and Epistatic eQTL Modules. PLOS Comp Bio 2010, 6(1):e1000642.

13. Brem RB, Kruglyak L: The landscape of genetic complexity across 5700 gene expression traits in yeast. Proc Natl Acad Sci USA 2005, 102(5):1572-1577.

14. Zhang B, Horvath S: A General Framework for Weighted Gene Co-Expression Newtork Analysis. Stat App/ Genet Molec Biol 2005, 4(1):Article 17.

15. Curtis RE, Xing EP: GenAMap: An Integrated Analytic and Visualization Platform for GWA and eQTL Analysis. Proceedings of the 17th International Conference on Intelligent Systems for Molecular Biology (ISMB) 2010. Technology Track.

16. Curtis RE, Kinnaird P, Xing EP: GenAMap: visualization strategies for association mapping. IEEE Symposium on Biological Data Visualization 2011, 1:87-95.

17. Yvert G, Brem RB, Whittle J, Akey JM, Foss E, et al: Trans-acting regulatory variation in Saccharomyces cerevisiae and the role of transcription factors. Nat Genet 2003, 35:57-64.

18. The Saccaromyces Genome Database:. [Online]. http://yeastgenome.org.

19. Chua G, Morris QD, Sopko R, Robinson MD, Ryan O, et al: Identifying transcription factor functions and targets by phenotypic activiation. PNAS 2006, 103(32):12045-12050.

20. Hughes TR, Marton MJ, Jones AR, Roberts CJ, Stoughton R, et al: Functional discovery via a compendium of expression profiles. Cell 2000, 102(1):109-126.

21. Maclssac KD, Wang T, Gordon DB, Gifford DK, Stormo GD, et al: An improved map of conserved regulatory sites for Saccharomyces cerevisiae. BMC Bioinformatics 2006, 7:113.

22. Zhu C, Byers KJ, McCord RP, Shi Z, Berger MF, et al: High-resolution DNAbinding specificity analysis of yeast transcription factors. Genome Res 2009, 19(4):556-566.

23. Harbison CT, Gordon DB, Lee TI, Rinaldi JN, Maclssac KD, et al: Transcriptional regulatory code of a eukaryotic genome. Nature 2004, 431:99-104.

24. Lee TI, Rinaldi NJ, Robert F, Odom DT, Bar-Joseph Z, et al: Transcriptional Regulatory Networks in Saccharomyces cerevisiae. Science 2002, 298(5594):799-804.

25. Brem RB, Storey JD, Whittle J, Kruglyak L: Genetic interactions between polymorphisms that affect gene expression in yeast. Nature 2005, 436(7051):701-703

26. Wei Z, Jun Z, EricE S, JunS L: A Bayesian Partition Method for Detecting Pleiotropic and Epistatic eQTL Modules. PLoS Comp Bio 2010, 6(1):e1000642.

27. Hughes JD, Estep PW, Tavazoie S, Church GM: Computational identification of cis-regulatory elements associated with groups of functionally related genes in Saccharomyces cerevisiae. J Mol Biol 2000, 296(5):1205-1214.

28. Sydorskyy Y, Dilworth DJ, Yi EC, Goodlett DR, Wozniak RW, et al: Intersection of the Kap123p-mediated nuclear import and ribosome export pathways. Mol Cell Biol 2003, 23(6):2042-2054

29. Cohn M, Blackburn EH: Telomerase in yeast. Science 1995, 269(5222):396-400.

30. Straatman KR, Louis EJ: Localization of telomeres and telomereassociation proteins in telomerase-negative Saccharomyces cerevisiae. Chromosome Res 2007, 15:1033-1050.

31. Yamada M, Hayatsu N, Matsuura A, Ishikawa F: Y'-Help1, a DNA helicase encoded by the yeast subtelomeric $Y^{\prime}$ element is induced in survivors defective for telomerase. J Biol Chem 1998, 273(50):33360-33366.

32. Dilworth DJ, Tackett AJ, Rogers RS, Yi EC, Christmas RH, et al: The mobile nucleoporin Nup2p and chromatin-bound Prp20p function in endogenous NPC-mediated transcriptional control. J Cell Biol 2005, 171(6):955-965.

33. Shore D: Telomere length regulation: getting the measure of chromosome ends. Biol Chem 1997, 387(7):591-597.

34. Teixeira MT, Arneric M, Sperisen P, Lingner J: Telomere length homeostasis is achieved via a switch between telomerase- extendable and nonextendable states. Cell 2004, 117(3):323-325.

35. Lee SI, Pe'er D, Dudley AM, Church GM, Koller D: Identifying regulator mechanisms using individual variation reveals key role for chromatin modification. PNAS 2006, 103(38):14062-14067.

36. Kim JM, Vanguri S, Boeke JD, Gabriel A, Voytas DF: Transposable elements and genome organization: a comprehensive survery of retrotransposons revealed by the complete Sacchararomyces cerevisiae genome sequence. Genome Res 1998, 8(5):464-478.

37. Boeke JD, Sandmeyer SB: "Yeast transposable elements,". In The molecular and cellular biology of the yeast Saccharomyces: genome dynamics, protein synthesis, and energetics. 1st edition. Edited by Broach JR, Jones EW, Pringle J. Cold Spring Harbor, New York: Cold Spring Harbor Laboratory Press; 1991:193-261.

38. Krastanova O, Hadzhitodorov M, Pesheva M: Ty Elements of the yeast Saccharomyces cerevisiae. Biotchnol Biotc Eq 2005, 19(3):19-26.
39. Curcio MJ, Hedge AM, Bocke JD, Garfinkel DJ: Ty RNA levels determine the spectrum of retrotranposition events that activate gene expression in Saccharomyces cerevisiae. Mol Gen Genet 1990, 220:213-221.

40. Kim S, Xing EP: Tree-guided group lasso for multi-task regression with structured sparsity. Proceedings of the 27th International Conference on Machine Learning (ICML) 2010

41. Puniyani K, Kim S, Xing EP: Multi-population GWA mapping via multi-taks regularized regression. Bioinformatics 2010, 26(12):i208-i216.

42. Lee S, Zhu J, Xing EP: Adaptive Multi-Task Lasso: with Application to eQTL Detection. In Advances in Neural Information Processing Systems 23 (NIPS). 2010 [http://www.proceedings.com/10901.html]

43. Curtis RE, Goyal A, Xing EP: Enhancing the usability and performance of structured association mapping algorithms using automation, parallelization, and visualization in the GenAMap software system. BMC Genet 2012, 13(24). doi:10.1186/1471-2156-13-24.

44. Luxburg U: A tutorial on spectral clustering. Statistics and Computing 2007, 17(4):395-416

45. Tibshirani R: Regression shrinkage and selection via the lasso. Royal Statist Soc B 1996, 58(1):267-288.

46. Saccharomyces cerevisiae RM11-1a sequencing project. [Online] http:// www.broadinstitute.org/annotation/genome/saccharomyces_cerevisiae/ Home.html.

47. NCBI. [Online] http://blast.ncbi.nlm.nih.gov/Blast.cgi.

48. King JY, Ferrara R, Tabibiazar R, SpinJ M, Chen MM, et al: Pathway analysis or coronary atherosclerosis. Physio/ Genomics 2005, 23(1):103-118.

\section{doi:10.1186/1471-2164-14-196}

Cite this article as: Curtis et al:: Structured association analysis leads to insight into Saccharomyces cerevisiae gene regulation by finding multiple contributing eQTL hotspots associated with functional gene modules. BMC Genomics 2013 14:196

\section{Submit your next manuscript to BioMed Central and take full advantage of:}

- Convenient online submission

- Thorough peer review

- No space constraints or color figure charges

- Immediate publication on acceptance

- Inclusion in PubMed, CAS, Scopus and Google Scholar

- Research which is freely available for redistribution
C Biomed Central 